University of San Diego

Digital USD

Biology: Faculty Scholarship

Department of Biology

$12-2018$

\title{
Polyglutamine repeat proteins disrupt actin structure in Drosophila photoreceptors.
}

\author{
Annie $\mathrm{Vu}$ \\ University of San Diego \\ Tyler Humphries \\ University of San Diego \\ Sean Vogel \\ University of San Diego \\ Adam Haberman \\ University of San Diego, ahaberman@sandiego.edu
}

Follow this and additional works at: https://digital.sandiego.edu/biology_facpub

Part of the Biology Commons, Cell Biology Commons, Laboratory and Basic Science Research

Commons, and the Molecular and Cellular Neuroscience Commons

\section{Digital USD Citation}

Vu, Annie; Humphries, Tyler; Vogel, Sean; and Haberman, Adam, "Polyglutamine repeat proteins disrupt actin structure in Drosophila photoreceptors." (2018). Biology: Faculty Scholarship. 30.

https://digital.sandiego.edu/biology_facpub/30

This Article is brought to you for free and open access by the Department of Biology at Digital USD. It has been accepted for inclusion in Biology: Faculty Scholarship by an authorized administrator of Digital USD. For more information, please contact digital@sandiego.edu. 


\title{
Polyglutamine repeat proteins disrupt actin structure in Drosophila photoreceptors.
}

\begin{abstract}
Expansions of polygutamine-encoding stretches in several genes cause neurodegenerative disorders including Huntington's Disease and Spinocerebellar Ataxia type 3. Expression of the human disease alleles in Drosophila melanogaster neurons recapitulates cellular features of these disorders, and has therefore been used to model the cell biology of these diseases. Here, we show that polyglutamine disease alleles expressed in Drosophila photoreceptors disrupt actin structure at rhabdomeres, as other groups have shown they do in Drosophila and mammalian dendrites. We show this actin regulatory pathway works through the small $\mathrm{G}$ protein Rac and the actin nucleating protein Form3. We also find that Form3 has additional functions in photoreceptors, and that loss of Form3 results in the specification of extra photoreceptors in the eye
\end{abstract}

\section{Keywords}

Actin, Drosophila, Formin, Huntington's disease, Photoreceptor, Polyglutamine

\section{Disciplines}

Biology | Cell Biology | Laboratory and Basic Science Research | Molecular and Cellular Neuroscience

\section{Notes}

Published in final form at:

Vu, Annie, Tyler Humphries, Sean Vogel, Adam Haberman. Polyglutamine repeat proteins disrupt actin structure in Drosophila photoreceptors. Molecular and Cellular Neuroscience, 93. 2018. doi: 10.1016/ j.mcn.2018.08.005.

\section{Creative Commons License}

\section{(c) (i)}

This work is licensed under a Creative Commons Attribution 4.0 License. 


\title{
Polyglutamine repeat proteins disrupt actin structure in Drosophila photoreceptors
}

\author{
Annie Vu, Tyler Humphries, Sean Vogel, Adam Haberman* \\ University of San Diego, Department of Biology, 5998 Alcala Park Blvd, SCST 372, San Diego, CA 92110, USA
}

\section{A R T I C L E I N F O}

\section{Keywords:}

Actin

Drosophila

Huntington's disease

Photoreceptor

Formin

Polyglutamine

\begin{abstract}
A B S T R A C T
Expansions of polygutamine-encoding stretches in several genes cause neurodegenerative disorders including Huntington's Disease and Spinocerebellar Ataxia type 3. Expression of the human disease alleles in Drosophila melanogaster neurons recapitulates cellular features of these disorders, and has therefore been used to model the cell biology of these diseases. Here, we show that polyglutamine disease alleles expressed in Drosophila photoreceptors disrupt actin structure at rhabdomeres, as other groups have shown they do in Drosophila and mammalian dendrites. We show this actin regulatory pathway works through the small G protein Rac and the actin nucleating protein Form3. We also find that Form3 has additional functions in photoreceptors, and that loss of Form 3 results in the specification of extra photoreceptors in the eye.
\end{abstract}

\section{Introduction}

Polyglutamine (polyQ) disorders are dominant neurodegenerative diseases caused by expanded CAG nucleotide tracts within protein coding genes (Orr and Zoghbi, 2007). There are nine known polyQ diseases, including Huntington's Disease (HD) and Spinocerebellar Ataxia type 3 (SCA3), each caused by CAG expansions encoding long stretches of glutamine within a different protein (HTT and ATXN3, respectively). In each case, having a small number of CAG repeats allows for wild type function of the encoded protein, while having a larger number of repeats causes a pathogenic gain of function and protein aggregation.

The fruit fly Drosophila melanogaster has been a powerful model system for investigating the cellular and molecular mechanisms of these diseases (McGurk et al., 2015). Researchers have expressed expanded, pathogenic alleles of polyQ genes in Drosophila neurons to study their cellular responses, while expressing short, wild type alleles as a control. Expressing human disease alleles in fly neurons causes defects that are similar to those seen in the corresponding disease (Jackson et al., 1998; McGurk et al., 2015; Warrick et al., 1998).

One feature common to polyQ diseases is reduced dendritic complexity (Clark et al., 1997; Graveland et al., 1985; Guidetti et al., 2001). Expression of HTT or ATXN3 disease alleles in Drosophila dendritic arborization neurons recapitulated these dendritic defects, which were caused by disruption of the actin cytoskeleton (Lee et al., 2011). Dendritic structure in these cells could be rescued by coexpression of an activated form of Rho-family GTPase Rac (Rac.V12), which regulates actin structure. Further understanding of interactions between polyQ proteins and the actin cytoskeleton would illuminate a poorly understood aspect of polyQ diseases.

Rho-family GTPases regulate actin filaments through direct interaction with Formins, actin nucleating proteins that promote the formation of linear actin filaments (Liu et al., 2010). Extensive studies of the formin diaphenous show that it exists in an auto-inhibited form until bound by activated Rho (Higgs, 2005). Drosophila melanogaster has six formin genes, any of which might be regulated by Rac in dendrites (Liu et al., 2010).

We have identified interactions between polyQ proteins and the actin cytoskeleton in Drosophila melanogaster photoreceptor neurons. These neurons are not required for life in a laboratory setting, allowing us to analyze cellular responses to polyQ proteins over time. Here we report that HTT and ATXN3 disease alleles induce massive disruptions of the actin cytoskeleton in photoreceptors, with severity that increases with age. Coexpression of Rac.V12 rescues these actin defects, demonstrating that the mechanism that patterns dendrites is also active in photoreceptors. We further show that formin Form 3 also regulates these actin structures, and may function downstream of Rac. We conclude that photoreceptors can be used to model dendritic actin regulation in response to polyQ proteins, as regulation of actin in photoreceptor rhabdomeres uses the same pathway employed in dendrites.

\footnotetext{
* Corresponding author.

E-mail address: ahaberman@sandiego.edu (A. Haberman).
} 

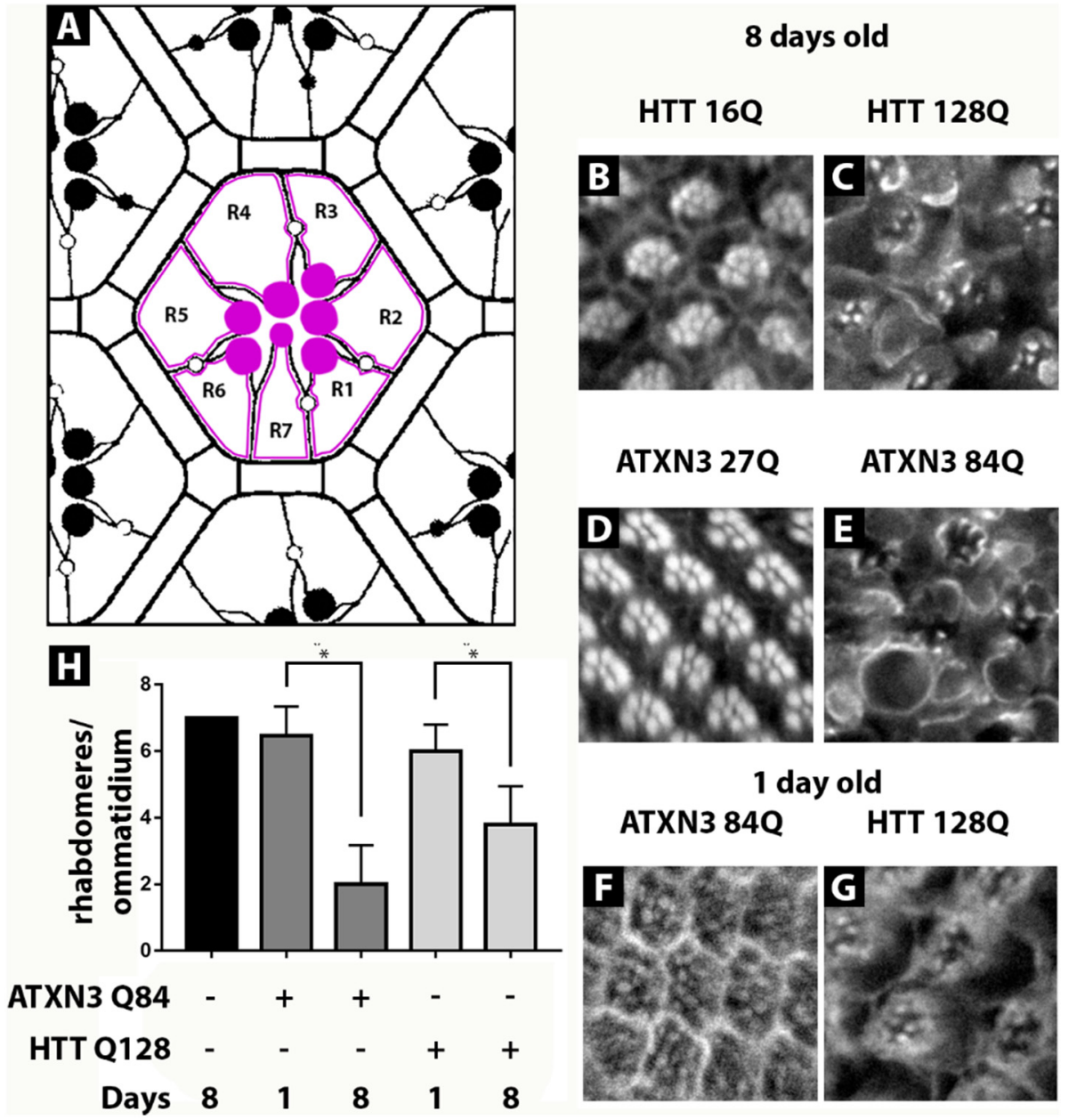

\section{8 days old}

HTT $16 Q$

\section{HTT 1280} 1 day old
ATXN3 840

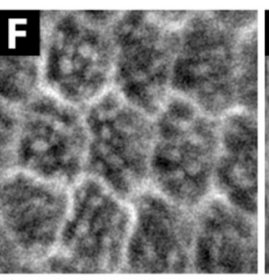

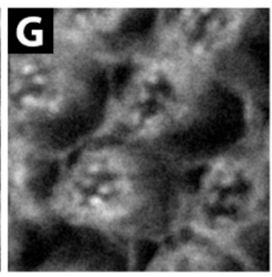

Fig. 1. Polyglutamine disease alleles disrupt actin organization in Drosophila melanogaster photoreceptors. A: Photoreceptors (R1-R7) accumulate actin (purple) at rhabdomeres and the cell cortex. B-E: Phalloidin staining reveals actin localization changes in photoreceptors expressing human polyglutamine genes under the control of GMR-Gal4 for 8 days. Short alleles of HTT (B) and ATXN3 (D) do not change wildtype actin localization within 8 days. Long, disease-associated alleles of HTT (C) and ATXN3 (E) cause a reduction of actin localization to rhabdomeres and increased localization to membranes. F-G: After only 1 day, actin is mislocalized less severely than at 8 days. $\mathrm{H}$ : Statistical analysis confirms a significant loss of rhabdomeres from day 1 to day $8 .{ }^{*}$ indicates $p<0.0001$. (For interpretation of the references to colour in this figure legend, the reader is referred to the web version of this article.)

\section{Materials and methods}

\subsection{Fly genetics}

Flies were raised at $25^{\circ} \mathrm{C}$ in alternating $12 \mathrm{~h}$ light and $12 \mathrm{~h}$ dark on Nutrifly BF media (Genesee Scientific, San Diego, CA). For all experiments, flies contained one copy of GMR-Gal4 and one copy of any UASregulated transgene. Fly strains GMR-Gal4 (Song et al., 2000), UASHTT.16Q, UAS-HTT.128Q (expressing full-length HTT) (Song et al., 2000), UAS-ATXN3.27Q, UAS-ATXN3.84Q (expressing full-length ATXN3) (Warrick et al., 2005), UAS-Rac.V12 (Luo et al., 1994), UASdia RNAi (P\{TRiP.HMS00308\}), UAS-capu RNAi (P\{TRiP.HMS00712\}), UAS-fhos RNAi (P\{TRiP.JF01606\}), UAS-frl RNAi (P \{TRiP.HMS00445\}), UAS-DAAM RNAi (P\{TRiP.HMS01978\}), UASform3 RNAi (P\{TRiP.HMS00393\}) (Ni et al., 2011) were obtained from the Bloomington Drosophila Stock Center.

\subsection{Eye staining}

Flies were collected once a day, and either dissected immediately ( 1 day old) or aged for 1 week before dissection ( 8 days old). Eyes were dissected, fixed, and stained as previously described (Williamson and Hiesinger, 2010). Eyes were stained with Texas Red-phalloidin (SigmaAldrich), phalloidin-CF555 (Biotium Inc., Feemont, CA), anti-Rh1 (4C5, Developmental Studies Hybridoma Bank, Iowa City, IA) and antimouse-Alexa 488 (VWR International, Radnor, PA) and mounted with Vectashield (Vector Laboratories, Burlingame, CA). Images were collected on a Nikon A1R confocal microscope (Nikon Instruments, Melville, NY) and processed with Fiji (Rueden et al., 2017; Schindelin et al.,
2012) and Photoshop CC 2015 (Adobe Systems Inc., San Jose, CA).

\subsection{Statistical analysis}

All statistical analyses were performed using GraphPad Prism 7.0. Rhabdomere areas from multiple genotypes were compared using one way ANOVA with Bonferroni multiple comparison procedures. 10 rhabdomeres from at least three different ommatidia were measured for each condition, using the polygon selection tool in Fiji to measure areas. Pairwise comparisons were performed using a two tailed $t$-test.

\section{Results}

\subsection{Polyglutamine-containing proteins disrupt photoreceptor actin structure}

Each facet, or ommatidium, of a Drosophila melanogaster eye contains 8 photoreceptor neurons (R1-R8), seven of which can be seen in a cross-section (Pellikka et al., 2002) (Fig. 1A). Outer photoreceptors (R1R6) are arranged in a stereotypical trapezoid pattern. Inner photoreceptors (R7 \& R8) are smaller and are stacked on one another vertically. All photoreceptors position a rhabdomere near the center of the ommatidium. The rhabdomere is made of tightly stacked plasma membrane containing high levels of the light-sensing transmembrane protein Rhodopsin. The rhabdomere also contains a high level of Factin. Phalloidin staining reveals the structure of the rhabdomeres, as well as cortical actin underlying the rest of the plasma membrane (Fig. 1A, purple).We expressed full-length human polyQ proteins in photoreceptor cells using GMR-Gal4, and analyzed actin structure by staining eyes with phalloidin. Eyes of eight day old flies expressing 


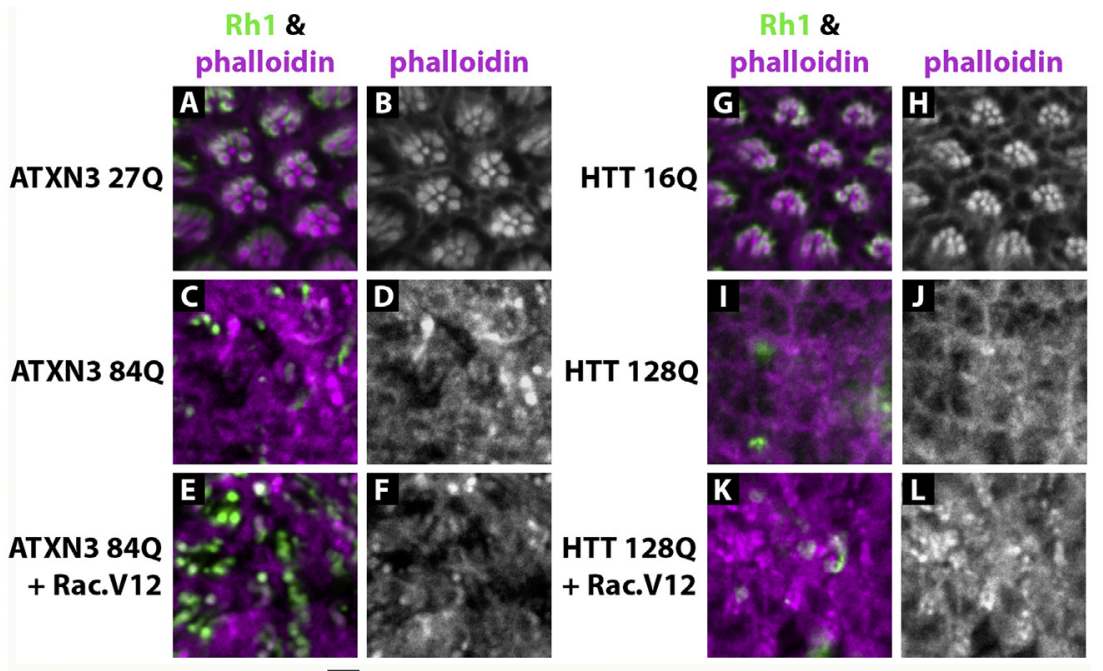

M
Fig. 2. Actin mislocalization is partially rescued by co-expression of activated Rac.V12. In I day old flies, Rh1 localizes to the six outer rhabdomeres in eyes expressing wild type alleles of ATXN3 (A, B) and HTT (G, H). Rhabdomeric actin and Rh1 accumulation are lost in eyes expressing diseases alleles of ATXN3 (C, D) and HTT (I, J). Co-expression of Rac.V12 restores actin localization to rhabdomeres for both disease alleles (F, L), but only restores $\mathrm{Rh} 1$ localization for ATXN3 (E, K). Mean number of actin-containing rhabdomeres and standard deviations are shown in $\mathrm{M}$. * indicates $p<0.05$. ** indicates $p<0.0001$.

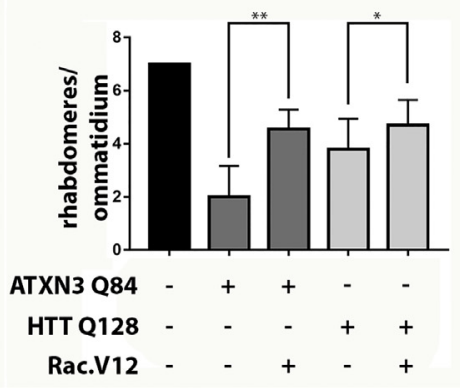

short, wild type forms of Huntingtin (HTT.16Q) or Ataxin 3 (ATXN3.27Q) in photoreceptors look wild type when stained with phalloidin (Fig. 1B, D), showing high levels of staining at rhabdomeres and lower levels at the cortex. The staining pattern was indistinguishable from that of eyes with GMR-Gal4 only (data not shown). Eyes expressing expanded, disease alleles of these genes (HTT.128Q and ATXN3. 84Q) dramatically change actin localization (Fig. 1C, E). Actin accumulates at some rhabdomeres, but these rhabdomeres are much smaller than wild type. Cortical accumulation of actin is enhanced and irregular, high in some regions and undetectable in others. In some ommatidia, rhabdomeres are not fully differentiated from the rest of the plasma membrane, and actin seems to accumulate equally on all of these membranes.

Since HD and SCA3 are degenerative disorders, whose severity increases over time, we wanted to see if polyQ-induced actin redistribution was also progressive. At 1 day of age, SCA3.84Q and HTT.128Q expressing eyes had more rhabdomeres and a more typical structure than at 8 days (Fig. 1F-H). SCA.84Q eyes had a mean of 6.5 rhabdomeres/ommatidium at day 1 compared to 2.0 at day 8 ( $t$-test, $p<0.0001$ ). HTT.128Q changed less dramatically, from 6.0 rhabdomeres/ommatidium at day 1 to 3.8 at day 8 ( $t$-test, $p<0.0001$ ). Rhabdomeres at day 1 were small, but clearly distinct from other membranes. F-actin also accumulated evenly at the cortex, though more abundantly than in wild type. We conclude that expanded polyQ proteins induce actin rearrangement, which becomes more severe and irregular over time.

3.2. Polyglutamine-induced photoreceptor defects are partially rescued by Rac signaling

To better characterize rhabdomere defects induced by long HTT and ATXN3 alleles, we stained eyes with both phalloidin and an antibody to
Rhodopsin (Rh1). Rh1 is the light detecting protein in outer photoreceptors, but is absent from inner photoreceptors. In eyes expressing HTT.16Q or ATXN3.27Q, Rh1 accumulates in its wild type pattern at the rhabdomere membrane (Fig. 2A, G). In eyes expressing HTT.128Q or ATXN3.84Q, Rh1 staining is mostly lost (Fig. 2C, I). Puncta of Rh1 still present in these eyes tend to colocalize with phalloidin puncta in remaining rhabdomeres.

PolyQ-induced actin mislocalization in dendritic arborization neurons was rescued by expression of a constitutively active form of the Rho-family GTPase Rac (Rac.V12) (Lee et al., 2011). To see if Rac also regulated actin in photoreceptors, we coexpressed expanded polyQ alleles and Rac.V12. Rac.V12 significantly rescued rhabdomeric accumulation of actin caused by both HTT.128Q and ATXN3.84Q, though the eyes were still disorganized (Fig. 2F, L, M). Interestingly, Rac.V12 rescued Rh1 accumulation in ATXN3.84Q-expressing photoreceptors, but not in HTT.128Q photoreceptors (Fig. 2E, K).

\subsection{Form 3 regulates actin accumulation in photoreceptors}

Small G proteins can regulate actin structure through formin proteins, which nucleate new actin filaments. Drosophila melanogaster has six formin orthologs: dia, capu, fhos, frl, DAAM, and form3 (Liu et al., 2010). To see which formins might function downstream of Rac in photoreceptors, we used RNAi to knock down each formin gene. Knockdown of most formin genes displayed only mild effects on eye development or actin structure in 1 day old eyes (Fig. 3A-E). Notably, frl knockdown caused ommatidial rotation defects, as previously reported (Fig. 3C) (Dollar et al., 2016). Only knockdown of fhos or form3 caused a reduction of rhabdomere size (ANOVA: $\mathrm{f}=42.62, \mathrm{df}=(6,63)$, $p<0.0001$ ) (Fig. 3E-G). Form3 knockdown also induced diffuse accumulation of f-actin throughout the photoreceptor cytoplasm. Therefore, form 3 appears to be a primary regulator of actin nucleation in 
dia RNAi

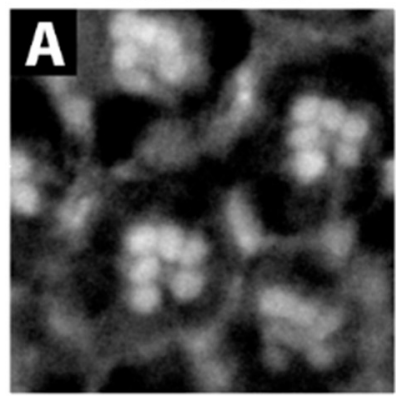

DAAM RNAi

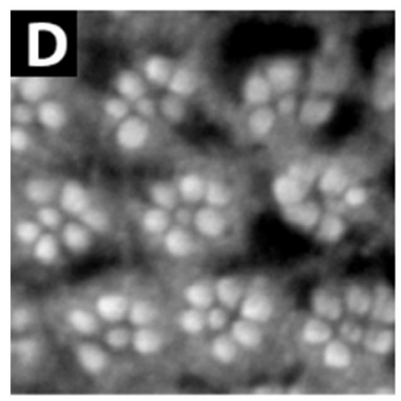

capu RNAi

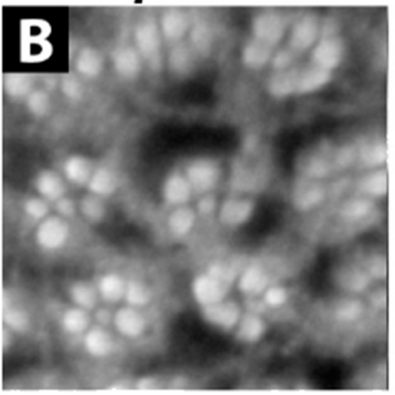

fhos RNAi

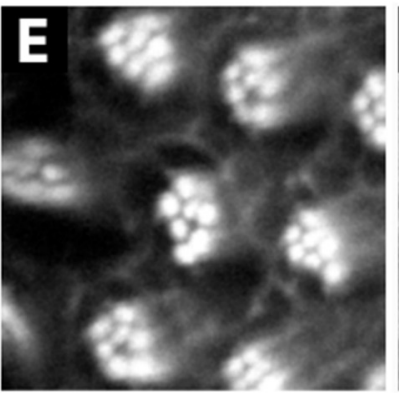

frl RNAi

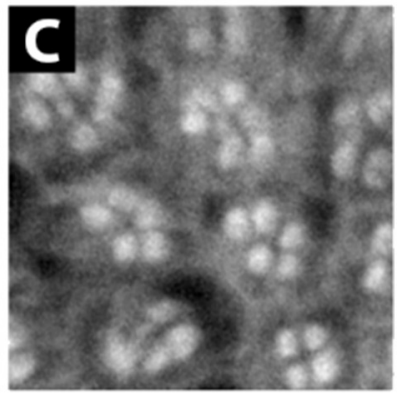

form3 RNAi

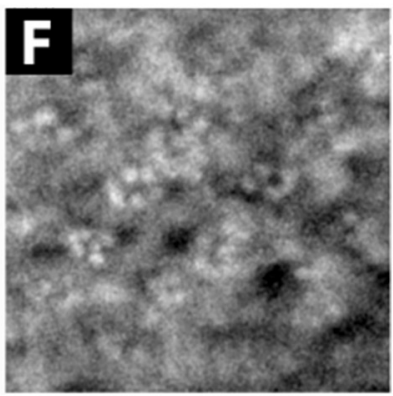

Fig. 3. Formins regulate actin structure in Drosophila photoreceptors. A-F: The six Drosophila formin genes were knocked down in photoreceptors using RNA interference. Knockdown of form 3 caused a loss of cortical actin, a strong reduction of rhabdomeric actin, and an accumulation of actin in the cytoplasm. G: Measurement of rhabdomere area shows that knockdown of fhos or form 3 reduced the size of the rhabdomere. Statistical analysis of rhabdomere areas shown in Table S1.
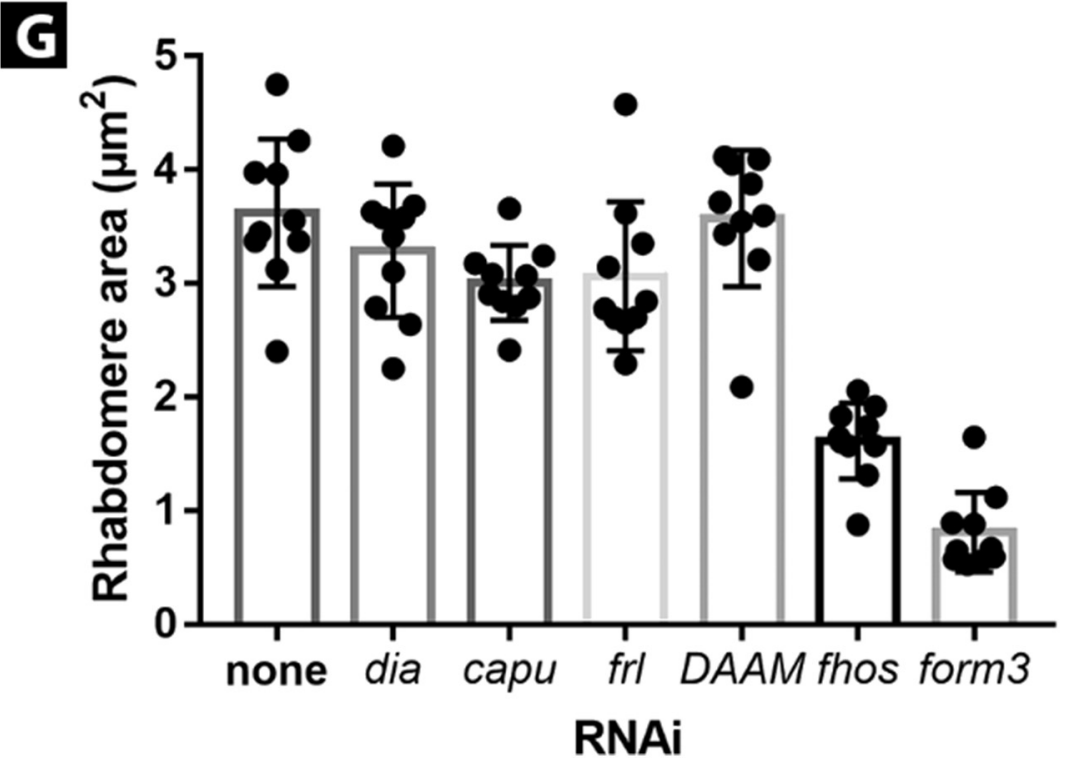

photoreceptor neurons, with actin forming aberrant structures in the absence of form3.

To see if form3 RNAi caused photoreceptor degeneration, we analyzed eyes of eight day old flies. Surprisingly, rhabdomeric and cortical actin was restored in older flies (Fig. 4B). Rhabdomere size was restored to wild type levels (ANOVA: $\mathrm{f}=105.5, \mathrm{df}=(2.27), p<0.0001$ ) (Fig. 4G). No sign of degeneration was observed.

If form3 functions downstream of Rac in photoreceptors, Rac.V12 should not rescue the form 3 RNAi actin defects. As predicted, form 3 RNAi flies co-expressing Rac.V12 were not significantly larger than form3 RNAi rhabdomeres and had diffuse cytoplasmic actin filaments (Fig. 4E, G).

\subsection{Form 3 regulates photoreceptor cell fate}

Eye development is regulated by a network of signaling pathways which ensure each ommatidium has 6 outer photoreceptors and 2 stacked inner photoreceptors. However, many ommatidia in form3 RNAi eyes had actin accumulations that looked like extra outer and inner photoreceptor rhabdomeres (Fig. 4B). Costaining with Rh1 antisera showed that these extra outer rhabdomeres strongly accumulated Rh1 in a typical rhabdomere pattern (Fig. 4A-C). Inner photoreceptor rhabdomeres also had weak accumulations of Rh1, which is not seen in wild type. Quantitation of this defect revealed that form3 RNAi ommatidia had an average of 7.7 Rh1-positive rhabdomeres compared to 6 in wild type ( $t$-test, $p<0.0001$ ).

Extra rhabdomeres are also apparent in form 3 RNAi, Rac.V12 eyes (Fig. 4D-F). All apparent outer photoreceptor rhabdomeres were positive for Rh1. Inner photoreceptor rhabdomeres did not accumulate Rh1, in contrast to eyes expressing form 3 RNAi alone. Therefore, Rac.V12 rescued some, but not all, of the defects caused by form3 RNAi. 


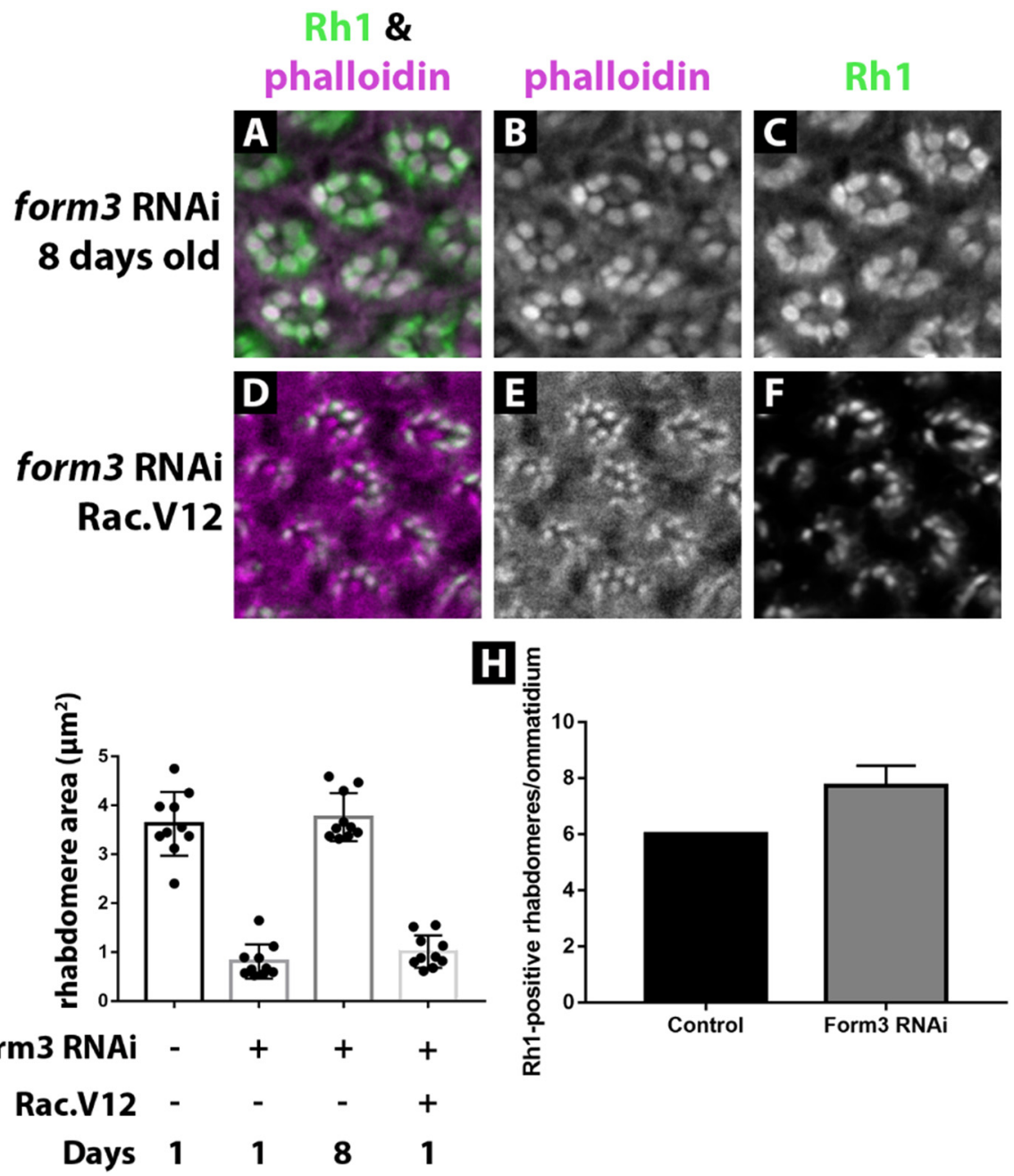

Fig. 4. Knockdown of form3 alters eye development. A-C: After 8 days of form 3 RNAi, actin structures have developed to wild type levels. Some ommatidia have additional large, outer photoreceptors with strong Rh1 staining. Others have an additional small, inner photoreceptor. The inner photoreceptors also express Rh1. D-F: Co-expression of Rac.V12 with form3 RNAi does not rescue actin defects at 1 day of age. Additional outer photoreceptors are seen in some ommatidia, but inner photoreceptors do not express Rh1 at this time point. G: Form3 RNAi rhabdomers at 1 day old are smaller than at 8 days old $(p<0.0001)$. H: Quantitation of additional Rh1-positive rhabdomeres in form3 RNAi eyes shows mean increase of 1.7 Rh1-positive rhabdomeres/ ommatidium $(p<0.0001)$.

\section{Discussion}

\subsection{Actin structure is disrupted by disease-associated polyQ proteins}

We have identified an actin regulatory pathway that is disrupted by disease-associated polyQ proteins in Drosophila neurons. While photoreceptors have rhabdomeres instead of the dendrites found in most neurons, both contain complex actin structures with analogous functions. Actin filaments are threaded though the membrane folds of the rhabdomere, providing structural support, and also form a web at the rhabdomere base (Arikawa et al., 1990) (Chang and Ready, 2000). Actin filaments also support dendritic structure, with actin disorganization resulting in reductions of dendritic size and complexity (Konietzny et al., 2017).

Therefore, we propose that rhabdomeric and dendritic defects caused by polyQ proteins are analogous, and that studies in photoreceptors can shed light on dendritic phenotypes. We report that activated Rac.V12 rescues rhabdomeric actin defects in photoreceptors, just as it rescues actin and dendrite defects in dendritic arborization neurons (Lee et al., 2011) (Fig. 2). This idea is supported by a recent yeast twohybrid screen that found HTT physically interacted with multiple small $\mathrm{G}$ protein regulatory proteins. Follow-up studies in mouse striatal cells found that Rac RNAi reduced the toxicity of expanded HTT proteins (Tourette et al., 2014). Thus the regulatory systems identified in fly photoreceptors may be relevant to mammalian disease processes.

We propose that actin accumulates at rhabdomeres and the cell cortex in a Form3-dependent manner, but that only rhabdomeric actin is regulated by Rac (Fig. 5A). Disease associated polyQ proteins disrupt the Rac-dependent pathway, resulting is less Form3 recruitment to rhabdomeres and leaving more Form 3 to associate with the cortex (Fig. 5B). This would result in the reduction of rhabdomeric actin and accumulation of cortical actin caused by polyQ proteins (Fig. 1). The partial rescue by Rac.V12 indicates that Rac-dependent signaling is only one of many processes disrupted by polyQ proteins (Fig. 2).

Disease associated polyQ proteins disrupt actin structures and dendrite morphology in many systems. In Drosophila dendritic arborization neurons, expanded polyQ alleles of HTT, ATXN1, and ATXN3 all reduce dendritic actin accumulation and cause a reduction in the size of the dendritic arbor (Lee et al., 2011). Expanded polyQ alleles of ATXN2 causes a loss of Purkinje cell dendritic arbor that precedes cell death (Pulst et al., 2000). Drosophila ATXN2 mutants have missing or disorganized actin structures in nurse cells, bristles, and developing eyes (Satterfield et al., 2002).

Loss of rhabdomeric actin increased as flies aged (Fig. 1). This progressive phenotype is reminiscent of the age-related progression of Huntington's Disease and Spinocerebellar Ataxia. However, the early, persistent expression of polyQ transgenes in fly eyes could also cause developmental defects that might alter this phenotype. In these studies, we are unable to separate any potential developmental defects from the progressive defects.

\subsection{Form3 regulates neuronal actin filaments}

Form3 regulates both rhabdomeric and cortical actin structures (Fig. 5C). We find that form3 RNAi causes reduced rhabdomeric actin and little detectable cortical actin in 1 day old photoreceptors (Fig. 3F). 
B

rhabdomeric actin
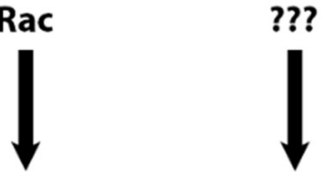

Form3

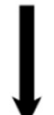

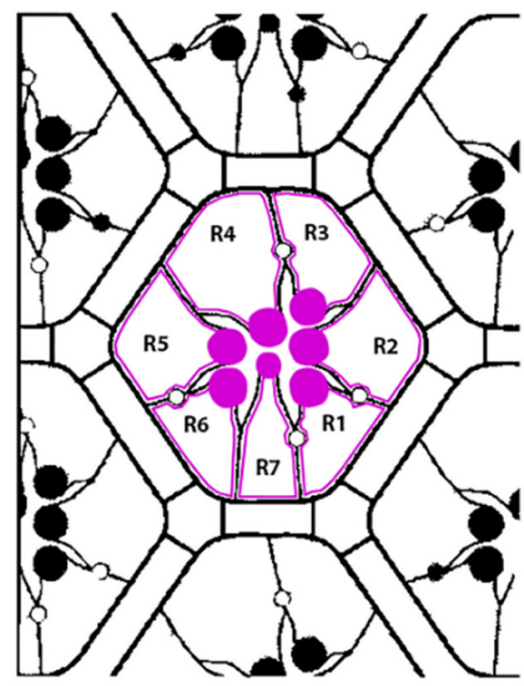

???

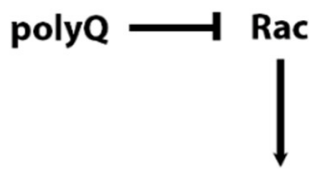

Form3

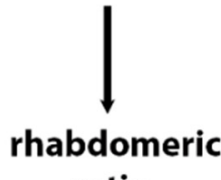

actin

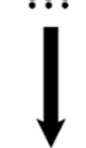

Form3

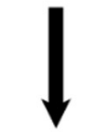

cortical

actin

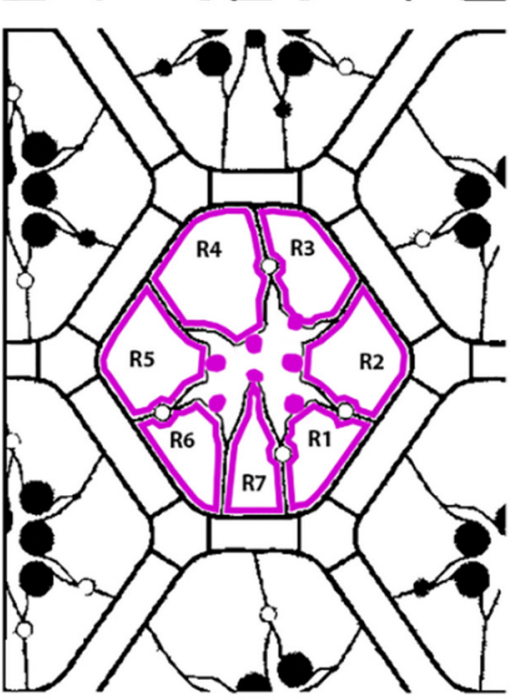

Fig. 5. Regulation of actin structures in Drosophila photoreceptors. A: In wild type eyes, actin localizes to both the rhabdomere and the cell cortex, under the control of Form3. Rac is responsible for Form3 activation at the rhabdomere, while unknown regulators direct cortical actin polymerization. B: Accumulation of disease-associated polyQ proteins disrupts Rac function at the rhabdomere, reducing rhabdomeric actin polymerization. More Form3 is available to function at the cortex, leading to increases cortical actin. C: Knockdown of form 3 reduces polymerization of actin at both the cortex and rhabdomere. Other actin-nucleating proteins induce actin polymerization in the cytoplasm, leading to diffuse actin accumulation.

$\mathbf{C}$

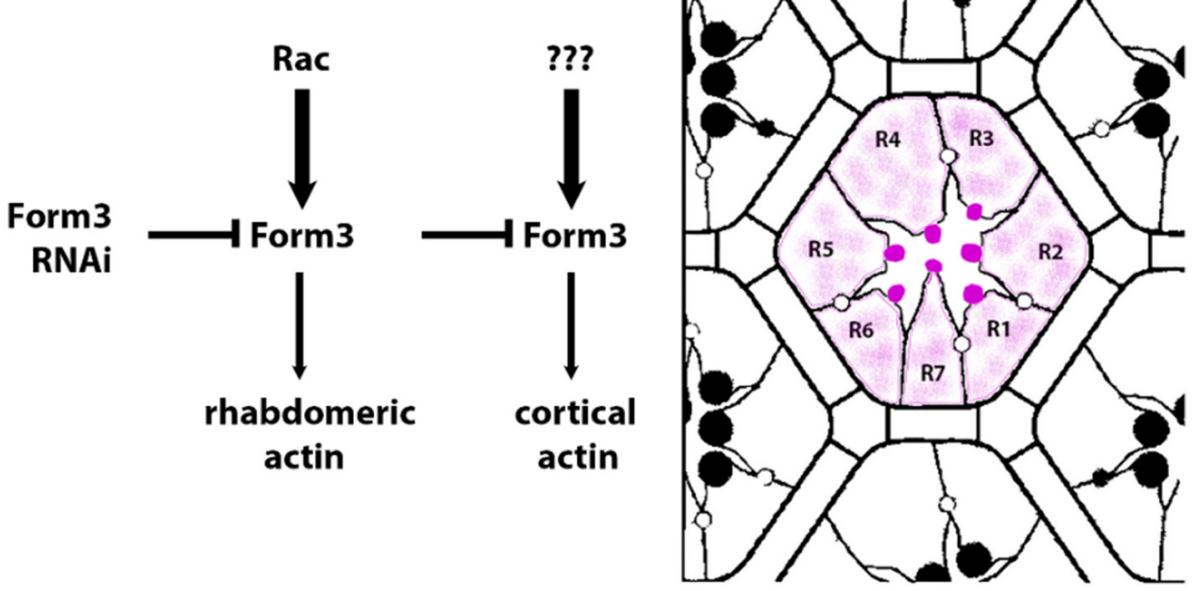

Instead, actin filaments assemble in the photoreceptor cytoplasm, presumably nucleated by other formins or other actin regulators. Other formins do function in photoreceptors. $\mathrm{Frl}$ is required for planar cell polarity in developing eyes, a requirement recapitulated in our studies (Dollar et al., 2016) (Fig. 3D). Double mutants of DAAM and Frl have stronger photoreceptor phenotypes than either mutant alone, indicating that DAAM is also active in these cells (Dollar et al., 2016). We found that fhos RNAi also affected rhabdomere size (Fig. 3E). Fhos has been shown to regulate actin in sarcomere formation and autophagic cell death, but has not previously been studied in the eye (Anhezini et al., 2012; Shwartz et al., 2016).

Form3 has not been extensively studied, but several reports indicate that it functions in Drosophila neurons. In developing embryos, form 3 is only expressed in tracheal and CNS cells (Tanaka et al., 2004). Form3 
RNAs were isolated from fly heads, but not from non-neuronal S2 cells (Ashwal-Fluss et al., 2014). Mutation of $t d p-43$ caused increased transcription of form3 in the larval CNS (Hazelett et al., 2012). RNAi of form 3 impaired the performance of flies in an olfactory assay (Walkinshaw et al., 2015). Thus, expression and functional data indicate that form 3 functions in many types of neurons.

\subsection{Form3 is required for eye development}

In addition to disrupting actin structures, form 3 RNAi altered eye development. Many ommatidia had extra outer and inner photoreceptors (Fig. 4B). Extra outer photoreceptors are also seen in fat facets ( faf) mutants, which fail to downregulate Notch signaling in developing eye discs (Overstreet et al., 2004). These extra photoreceptors are cells that would otherwise have developed as cone cells. Like faf mutants, form3 RNAi causes a rough eye phenotype (data not shown). It is unclear if Notch signaling is involved in the form 3 RNAi phenotype.

Extra inner photoreceptor might also be due to changes in cell fate, or might be due to a change in inner photoreceptor morphology. Normally, the two inner photoreceptors are stacked and only one can be seen in a focal plane. A change in the growth or organization of these cells might cause them to lie next to one another, so that both could be seen in a single image. Notably, we never detected more than two inner photoreceptors in an ommatium.

Form3 RNAi inner photoreceptors also expressed low levels of Rh1, which does not occur in wild type inner photoreceptors (Fig. 4C). This change in gene expression is surprising, as there is no clear mechanism for a formin to impact gene expression. Coexpression of Rac.V12 with form3 RNAi rescues this Rh1 phenotype without impacting the actin phenotype, suggesting that Rh1 expression is not just responding to the change in actin regulation (Fig. 4F). Therefore, form 3 may have functions beyond actin regulation in photoreceptor development.

\section{Conclusions}

We have found that expanded PolyQ protein disrupt a neuronal actin regulatory pathway involving Rac and Form3. While our work was performed in Drosophila photoreceptor neurons, it is in agreement with studies in other neurons and other species. Given the practical advantages of photoreceptors for studies of neurodegeneration, we propose that photoreceptors will be a useful system for further studies of this pathway. We have also identified Form3 as an actin regulator in Drosophila neurons, and shown that it is required for proper eye development.

Supplementary data to this article can be found online at https:// doi.org/10.1016/j.mcn.2018.08.005.

\section{Acknowledgements}

The authors gratefully acknowledge Joseph Provost, Kate Boersma, Jillian Wothe, Biol 480L students, and two anonymous reviewers for advice and critiques on the manuscript. Stocks obtained from the Bloomington Drosophila Stock Center (NIH P40OD018537) were used in this study. The anti-Rh1 monoclonal antibody developed by de Couet and Tanimura was obtained from the Developmental Studies Hybridoma Bank, created by the NICHD of the NIH and maintained at The University of Iowa, Department of Biology, Iowa City, IA 52242. This research was supported by a Faculty Research Grant and an Associated Students Grant from the University of San Diego.

\section{References}

Anhezini, L., Saita, A.P., Costa, M.S.A., Ramos, R.G.P., Simon, C.R., 2012. Fhos encodes a Drosophila Formin-Like Protein participating in autophagic programmed cell death. Genesis 50, 672-684. https://doi.org/10.1002/dvg.22025.

Arikawa, K., Hicks, J.L., Williams, D.S., 1990. Identification of actin filaments in the rhabdomeral microvilli of Drosophila photoreceptors. J. Cell Biol. 110, 1993-1998. Ashwal-Fluss, R., Meyer, M., Pamudurti, N.R., Ivanov, A., Bartok, O., Hanan, M., Evantal, N., Memczak, S., Rajewsky, N., Kadener, S., 2014. CircRNA biogenesis competes with pre-mRNA splicing. Mol. Cell 56, 55-66. https://doi.org/10.1016/j.molcel.2014.08. 019.

Chang, H.Y., Ready, D.F., 2000. Rescue of photoreceptor degeneration in rhodopsin-null Drosophila mutants by activated Rac1. Science 290, 1978-1980. https://doi.org/10. 1126/science.290.5498.1978. (80-. ).

Clark, H.B., Burright, E.N., Yunis, W.S., Larson, S., Wilcox, C., Hartman, B., Matilla, A., Zoghbi, H.Y., Orr, H.T., 1997. Purkinje cell expression of a mutant allele of SCA1 in transgenic mice leads to disparate effects on motor behaviors, followed by a progressive cerebellar dysfunction and histological alterations. J. Neurosci. 17, 7385-7395.

Dollar, G., Gombos, R., Barnett, A.A., Hernandez, D.S., Maung, S.M.T., Mihly, J., Jenny, A., 2016. Unique and overlapping functions of formins frl and DAAM during ommatidial rotation and neuronal development in Drosophila. Genetics 202, 1135-1151. https://doi.org/10.1534/genetics.115.181438.

Graveland, G., Williams, R., Difiglia, M., 1985. Evidence for degenerative and regenerative changes in neostriatal spiny neurons in Huntington's disease. Science 227 (80-. ).

Guidetti, P., Charles, V., Chen, E.-Y., Reddy, P.H., Kordower, J.H., Whetsell, W.O., Schwarcz, R., Tagle, D.A., 2001. Early degenerative changes in transgenic mice expressing mutant huntingtin involve dendritic abnormalities but no impairment of mitochondrial energy production. Exp. Neurol. 169, 340-350. https://doi.org/10. 1006/exnr.2000.7626.

Hazelett, D.J., Chang, J.-C., Lakeland, D.L., Morton, D.B., 2012. Comparison of parallel high-throughput RNA sequencing between knockout of TDP-43 and its overexpression reveals primarily nonreciprocal and nonoverlapping gene expression changes in the central nervous system of Drosophila. G3 (Bethesda) 2, 789-802. https://doi.org/10.1534/g3.112.002998.

Higgs, H.N., 2005. Formin proteins: a domain-based approach. Trends Biochem. Sci. 30, 342-353. https://doi.org/10.1016/J.TIBS.2005.04.014.

Jackson, G.R., Salecker, I., Dong, X., Yao, X., Arnheim, N., Faber, P.W., MacDonald, M.E., Zipursky, S.L., 1998. Polyglutamine-expanded human huntingtin transgenes induce degeneration of Drosophila photoreceptor neurons. Neuron 21, 633-642. https://doi. org/10.1016/S0896-6273(00)80573-5.

Konietzny, A., Bär, J., Mikhaylova, M., 2017. Dendritic actin cytoskeleton: structure, functions, and regulations. Front. Cell. Neurosci. 11, 147. https://doi.org/10.3389/ fncel.2017.00147.

Lee, S.B., Bagley, J.a., Lee, H.Y., Jan, L.Y., Jan, Y.-N.Y.-N., 2011. Pathogenic polyglutamine proteins cause dendrite defects associated with specific actin cytoskeletal alterations in Drosophila. Proc. Natl. Acad. Sci. U. S. A. 108, 16795-16800. https:// doi.org/10.1073/pnas.1113573108.

Liu, R., Linardopoulou, E.V., Osborn, G.E., Parkhurst, S.M., 2010. Formins in development: orchestrating body plan origami. Biochim. Biophys. Acta, Mol. Cell Res. 1803, 207-225. https://doi.org/10.1016/j.bbamcr.2008.09.016.

Luo, L., Liao, Y.J., Jan, L.Y., Jan, Y.N., 1994. Distinct morphogenetic functions of similar small GTPases: Drosophila Drac1 is involved in axonal outgrowth and myoblast fusion. Genes Dev. 8, 1787-1802.

McGurk, L., Berson, A., Bonini, N.M., 2015. Drosophila as an in vivo model for human neurodegenerative disease. Genetics 201, 377-402. https://doi.org/10.1534/ genetics.115.179457.

Ni, J.-Q., Zhou, R., Czech, B., Liu, L.-P., Holderbaum, L., Yang-Zhou, D., Shim, H.-S., Tao, R., Handler, D., Karpowicz, P., Binari, R., Booker, M., Brennecke, J., Perkins, L.A., Hannon, G.J., Perrimon, N., 2011. A genome-scale shRNA resource for transgenic RNAi in Drosophila. Nat. Methods 8, 405-407. https://doi.org/10.1038/nmeth.1592.

Orr, H.T., Zoghbi, H.Y., 2007. Trinucleotide repeat disorders. Annu. Rev. Neurosci. 30, 575-621. https://doi.org/10.1146/annurev.neuro.29.051605.113042.

Overstreet, E., Fitch, E., Fischer, J.A., 2004. Fat facets and liquid facets promote Delta endocytosis and Delta signaling in the signaling cells. Development 131, 5355-5366. https://doi.org/10.1242/dev.01434.

Pellikka, M., Tanentzapf, G., Pinto, M., Smith, C., Mcglade, J., Ready, D., Tepass, U., 2002. Crumbs, the Drosophila homologue of human CRB1/RP12, is essential for photoreceptor morphogenesis. Nature 416, 143-149. https://doi.org/10.1038/ nature721.

Pulst, S.-M., Huynh, D.P., Figueroa, K., Hoang, N., 2000. Nuclear localization or inclusion body formation of ataxin-2 are not necessary for SCA2 pathogenesis in mouse or human. Nat. Genet. 26, 44-50. https://doi.org/10.1038/79162.

Rueden, C.T., Schindelin, J., Hiner, M.C., DeZonia, B.E., Walter, A.E., Arena, E.T., Eliceiri, K.W., 2017. ImageJ2: ImageJ for the next generation of scientific image data. BMC Bioinf. 18, 529. https://doi.org/10.1186/s12859-017-1934-z.

Satterfield, T.F., Jackson, S.M., Pallanck, L.J., 2002. A Drosophila homolog of the polyglutamine disease gene SCA2 is a dosage-sensitive regulator of actin filament formation. Genetics 162, 1687-1702.

Schindelin, J., Arganda-Carreras, I., Frise, E., Kaynig, V., Longair, M., Pietzsch, T., Preibisch, S., Rueden, C., Saalfeld, S., Schmid, B., Tinevez, J.-Y., White, D.J., Hartenstein, V., Eliceiri, K., Tomancak, P., Cardona, A., 2012. Fiji: an open-source platform for biological-image analysis. Nat. Methods 9, 676-682. https://doi.org/10. 1038/nmeth.2019.

Shwartz, A., Dhanyasi, N., Schejter, E.D., Shilo, B.-Z., 2016. The Drosophila formin Fhos is a primary mediator of sarcomeric thin-filament array assembly. elife 5. https://doi. org/10.7554/eLife.16540.

Song, Z., Guan, B., Bergman, A., Nicholson, D.W., Thornberry, N.A., Peterson, E.P., Steller, H., 2000. Biochemical and genetic interactions between Drosophila caspases and the proapoptotic genes rpr, hid, and grim. Mol. Cell. Biol. 20, 2907-2914.

Tanaka, H., Takasu, E., Aigaki, T., Kato, K., Hayashi, S., Nose, A., 2004. Formin3 is 
required for assembly of the F-actin structure that mediates tracheal fusion in Drosophila. Dev. Biol. 274, 413-425. https://doi.org/10.1016/j.ydbio.2004.07.035. Tourette, C., Li, B., Bell, R., Hare, S.O., Kaltenbach, L.S., Mooney, S.D., Hughes, R.E., 2014. A Large Scale Huntingtin Protein Interaction Network Implicates Rho GTPase Signaling Pathways in Huntington. 289. pp. 6709-6726. https://doi.org/10.1074/ jbc.M113.523696.

Walkinshaw, E., Gai, Y., Farkas, C., Richter, D., Nicholas, E., Keleman, K., Davis, R.L., 2015. Identification of genes that promote or inhibit olfactory memory formation in Drosophila. Genetics 199, 1173-1182. https://doi.org/10.1534/genetics.114.173575.
Warrick, J.M., Paulson, H.L., Gray-Board, G.L., Bui, Q.T., Fischbeck, K.H., Pittman, R.N., Bonini, N.M., 1998. Expanded polyglutamine protein forms nuclear inclusions and causes neural degeneration in Drosophila. Cell 93, 939-949.

Warrick, J.M., Morabito, L.M., Bilen, J., Gordesky-Gold, B., Faust, L.Z., Paulson, H.L., Bonini, N.M., 2005. Ataxin-3 suppresses polyglutamine neurodegeneration in Drosophila by a ubiquitin-associated mechanism. Mol. Cell 18, 37-48. https://doi. org/10.1016/j.molcel.2005.02.030.

Williamson, W.R., Hiesinger, P.R., 2010. Preparation of developing and adult Drosophila brains and retinae for live imaging. J. Vis. Exp. https://doi.org/10.3791/1936. 